\title{
Phase Diagram and Structural Diversity of a Family of Truncated Cubes: Degenerate Close-Packed Structures and Vacancy-Rich States
}

\author{
Anjan P. Gantapara, ${ }^{1, *}$ Joost de Graaf, ${ }^{1}$ René van Roij, ${ }^{2}$ and Marjolein Dijkstra ${ }^{1, \dagger}$ \\ ${ }^{1}$ Soft Condensed Matter, Debye Institute for Nanomaterials Science, Utrecht University, \\ Princetonplein 5, 3584 CC Utrecht, Netherlands \\ ${ }^{2}$ Institute for Theoretical Physics, Utrecht University, Leuvenlaan 4, 3584 CE Utrecht, Netherlands
}

(Received 1 March 2013; published 1 July 2013)

\begin{abstract}
Using Monte Carlo simulations and free-energy calculations, we determine the phase diagram of a family of truncated hard cubes, where the shape evolves smoothly from a cube via a cuboctahedron to an octahedron. A remarkable diversity in crystal phases and close-packed structures is found, including a fully degenerate crystal structure, several plastic crystals, as well as vacancy-stabilized crystal phases, all depending sensitively on the precise particle shape. Our results illustrate the intricate relation between phase behavior and building-block shape, and can guide future experimental studies on polyhedral-shaped nanoparticles.
\end{abstract}

PACS numbers: 61.46.Df, 64.70.M-, 64.75.Yz, 82.70.Dd

Recent advances in experimental techniques to synthesize polyhedron-shaped particles, such as faceted nanocrystals and colloids [1-14] and the ability to perform self-assembly experiments with these particles [15-22], have attracted the interest of physicists, mathematicians, and computer scientists [23-27]. Additionally, predicting the densest packings of hard polyhedra has intrigued mathematicians since the time of the early Greek philosophers, such as Plato and Archimedes [28,29]. Modern computer platforms have made it possible to perform simulations of these systems, which has resulted not only in an improved understanding of the experimentally observed phenomenology in colloidal suspensions of such particles, but also in improved Ansätze for the morphology of their closepacked configurations [24,30-35].

The self-assembly of the basic building blocks at finite pressures may differ substantially from the packings achieved at high (sedimentation and solvent-evaporation) pressures. For instance, liquid-crystal, plastic-crystal, vacancy-rich simple-cubic, and quasicrystalline mesophases are stabilized by entropy alone under non-closepacked conditions of hard anisotropic particle systems [30-34,36,37]. Predicting the phase behavior from the shape of the building blocks alone is therefore a major challenge in materials science and is crucial for the design of new functional materials. It is thus not surprising that numerous studies have been devoted to providing simple guidelines for predicting the self-assembly from the particle shape alone [32-34].

Recently, Henzie et al. [15] reported the shapecontrolled synthesis of truncated cubes. In their research, the close-packed crystals of these particles were studied using sedimentation experiments and simulations. They created exotic superlattices, and their results also tested several conjectures on the densest packings of hard polyhedra [23,25-27]. However, Henzie et al. did not examine the finite-pressure behavior of the system. Mapping the full phase diagram for the system of truncated cubes is thus important, not only from a fundamental perspective but also to guide future experimental self-assembly studies to fabricate new functional materials with these building blocks.

In this Letter, we investigate the equilibrium phase diagram of a family of truncated hard cubes, which interpolates smoothly between cubes and octahedra via cuboctahedra. Our calculations show that the phase diagram exhibits a remarkably rich diversity in crystal structures that depends sensitively on the particle shape. We find distinct changes in phase behavior and crystal structures even for small variations in the level of truncation. This is an unexpected result, since the particle shape varies smoothly from that of a cube to that of an octahedron by truncation. Moreover, we find that the equilibrium concentration of vacancies, which is already unusually high for a simple-cubic phase of cubes [36], increases at a fixed packing fraction $\phi$ upon increasing the level of truncation. In this Letter, we identify and describe in detail the different phases that we obtained, as well as the nature of the phase transitions between these phases.

The particles that we investigate are completely specified by the level of truncation $s \in[0,1]$ and the volume of the particle; see Fig. 1(a) and the Supplemental Material [38] for the definition of the truncated cube and additional details. Two Platonic (cube and octahedron) and three Archimedean (truncated cube, cuboctahedron, and truncated octahedron) solids are members of this family.

Using the floppy-box Monte Carlo method [39-42] in combination with a separating-axis-based overlap algorithm [43], we first numerically determined the densest structure and the corresponding packing fraction; see Fig. 1(b). Note that the packing fraction "curve" is continuous but has discontinuities in its first derivative. Cubes 
(a)

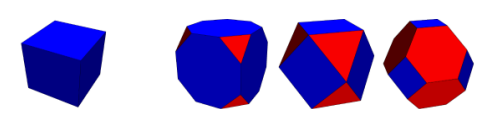

(b)

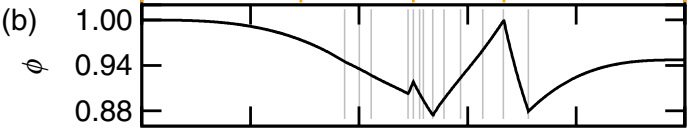

(c) $1.20-$

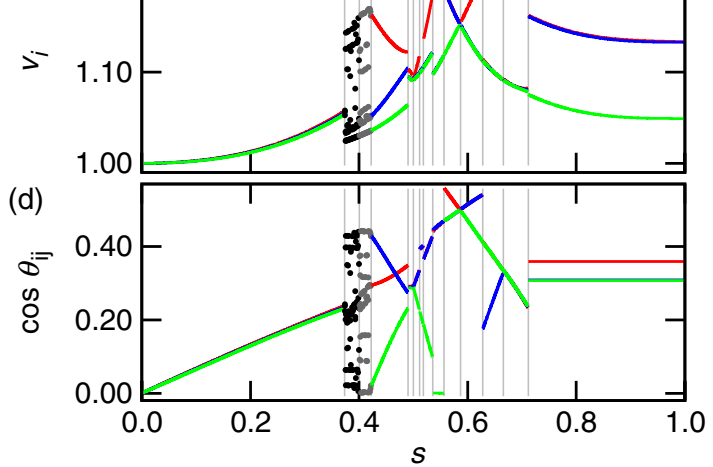

FIG. 1 (color online). (a) Five truncated cubes (Platonic and Archimedean solids only) for levels of truncation $s$ corresponding to the orange lines from left to right: a cube, a truncated cube, a cuboctahedron, a truncated octahedron, and an octahedron. (b) The packing fraction $\phi$ for the close-packed structures as a function of $s$. (c) The length $v_{i}(i=1,2,3)$ of the three lattice vectors, indicated in red, green, and blue, that span the unit cell of the densest crystal structure. Not every line is clearly visible, since there is some overlap. Black and gray dots are used in regions with a degeneracy in the crystal structures. (d) The cosine of the angles $\theta_{i j}(i<j=1,2,3)$ between the three $v_{i}$ in (b). Gray vertical lines partition the $s$ domain into 14 pieces with a "different" crystal structure, based on the discontinuities of $v_{i}$ and $\cos \theta_{i j}$.

$(s=0)$ and truncated octahedra $(s=2 / 3)$ are the only shapes that have space-filling close-packed structures in the entire $s \in[0,1]$ domain. We analyze our results by considering the lengths $v_{i}$ of and the angles $\theta_{i j}(i<j=1$, 2,3 ) between the three lattice vectors that specify the unit cell of the densest crystal structure; these quantities are shown in Figs. 1(c) and 1(d), respectively. Numerical analysis of the discontinuities in the (first derivative of the) $\phi, v_{i}$, and $\cos \theta_{i j}$ curves allows us to partition the $s \in[0,1]$ domain into 14 distinct regions.

We only briefly discuss the different close-packed structures here, and we refer the interested reader to the Supplemental Material [38] for visual representations and more detailed descriptions. We find (distorted) simple-cubic [(d)sc] phases for $s \in[0.00,0.374]$. For $s \in(0.374,0.40]$ and $s \in(0.40,0.422]$, we observe a large scatter in the lattice vectors, which we expect to approach a continuous spectrum for a larger number of simulations. This corresponds to a degeneracy in the densest crystal structures. The truncated cubes are arranged in a dsc crystal lattice, where the particles form columns that are interlocked in a diagonal way. This prevents lateral motion in the plane normal to the column's direction but allows motion in the direction of the columns for the diagonally interlocked sheets; see Ref. [38] for a visual representation. These structures are referred to as monointerlocking distorted simple-cubic (MI dsc) crystals. For $s \in(0.422,0.49]$, a dsc crystal phase is found that is interlocking in two directions (BI dsc), while for $s \in(0.49,0.50)$, a tri-interlocking dsc crystal (TI dsc) is observed. Multiple instances of interlocking prevent motion in the crystal, as also follows from the unicity of the results in these regions in Figs. 1(c) and 1(d). For $s \in[0.50,0.54]$ and $s \in(0.63,0.71]$, three different distorted bodycentered-tetragonal (dbct) structures are found, and for $s \in(0.54,0.63]$, there is a regular body-centered-tetragonal (bct) structure. Finally, for $s \in(0.71,1.00]$, we observe the Minkowski crystal phase. We thus find a remarkable diversity in close-packed structures that depends sensitively on the level of truncation. Below, we investigate the repercussions of the 11 distinct close-packed structures on the behavior at finite pressure.

Using the floppy-box Monte Carlo method results in combination with regular isothermal-isobaric (NPT) simulations and free-energy calculations, we are able to establish the phase diagram for hard truncated cubes, as shown in Fig. 2. For $s<0.5$, the particles are essentially "cubic" in shape, and we find high-density simple-cubic-like phases. The phase diagram for truncated cubes with shape parameter $s \in[0.00,0.35]$ displays three stable bulk phases. At very high pressures, we observe a dsc crystal phase similar to the $C 1$ phase of Ref. [30]. This phase melts either via a weak first-order or via a second-order phase transition into a vacancy-rich simple-cubic (sc) crystal phase. At even lower pressures, the sc crystal is found to coexist with the fluid phase. For $s \in(0.35,0.422]$, the phase diagram exhibits four stable phases separated by three two-phase coexistence regions. At sufficiently high pressures, the systems self-assemble into their respective densest-packed structures, which is the dsc $(C 1)$ structure for $s \in(0.35,0.374]$ and the MI dsc phase for $s \in$ $(0.374,0.422]$. Upon lowering the pressure, a first-order transition occurs to the dsc phase with the $\mathrm{C} 0$-like morphology of Ref. [30]. By further lowering the pressure, the dsc $(C 0)$ phase melts in all our simulations into a plastic hexagonal close-packed (phcp) crystal structure, before finally melting into a fluid phase. We find a dsc $(C 0)$ phcp-liquid triple point at $s \approx 0.374$. For $s \in(0.422,0.5]$, we observe higher orders of interlocking of the dsc crystal phase at sufficiently high pressures: a BI dsc and a TI dsc crystal, respectively. These phases melt into the phcp phase and subsequently into the isotropic liquid phase upon lowering the pressure, again via first-order phase transitions in both instances. Large lattice vector fluctuations and the degeneracy of the respective crystal structures prohibit free-energy calculations for $s \in[0.35,0.5]$.

For $s>0.5$ the shape is "octahedronlike," and we find bct-like structures at close packing. For $s \in[0.5,0.54]$, the 


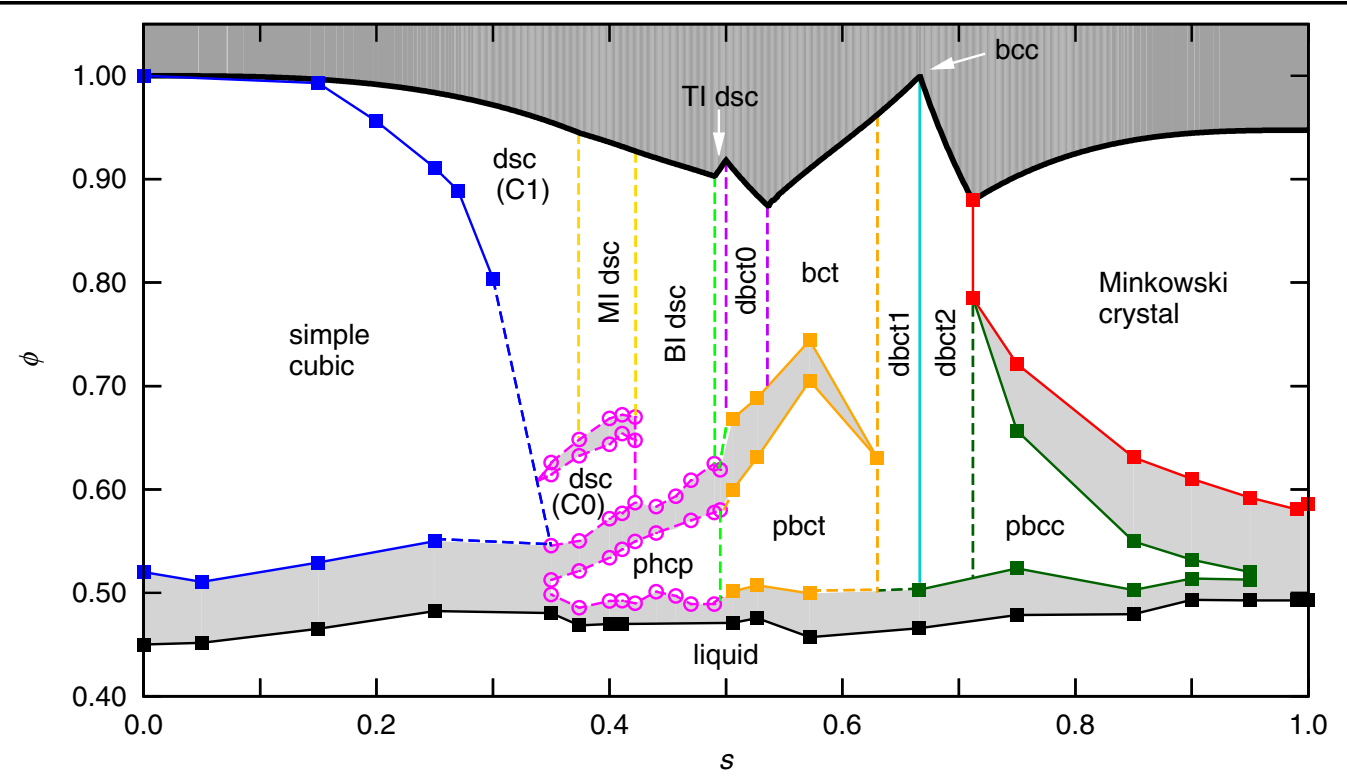

FIG. 2 (color online). Phase diagram for the family of truncated hard cubes in the packing fraction $\phi$ versus the level of truncation $s$ representation. In the dark-gray area, $\phi$ exceeds the maximum packing fraction. The light-gray areas indicate the two-phase coexistence regions. The solid square symbols denote the bulk coexistence densities as obtained from free-energy calculations, while the open circles indicate those derived from the equations of state. Coexistence lines that follow from free-energy calculations are represented by solid lines, and those that connect equation-of-state-derived points are given by dashed lines. The numbers that follow the dbct label signify that these dbct phases are distinct. The two dsc phases have different morphologies: one is $C 0$-like, and the other is $C 1$-like. Finally, the two white arrows in the forbidden region connect the label TI dsc to the small region between the green and purple dashed lines and the label bcc with the turquoise line, respectively.

close-packed dbct phase melts into a plastic bct (pbct) phase upon lowering the pressure via a first-order phase transition. At even lower pressures, we find two-phase coexistence between the pbct and the fluid phase. Interestingly, although the particles in this pbct phase rotate almost freely, their orientational distribution is not isotropic, which gives rise to the pbct rather than a plastic body-centered-cubic (pbcc) phase; see the movie in the Supplemental Material [38]. In the region $s \in$ $(0.54,0.636]$, we obtain a regular bct phase at high pressures, which undergoes a first-order transition into the pbct phase for intermediate pressures. For $s \in(0.636,0.712]$, we find two different dbct crystal structures (dbct1 and dbct2 in Fig. 2). The transition between dbct 1 and dbct 2 is located at $s=2 / 3$ (the space-filling truncated octahedron of which all sides are of equal length). Remarkably, this system exhibits a body-centered-cubic (bcc) crystal structure, which exists only for this exact value of the truncation $s=2 / 3$. Surprisingly, the close-packed crystal structures in the region $s \in(0.636,0.712]$ melt directly into a liquid phase via a first-order phase transition upon decreasing the pressure without an intervening plastic-crystal phase. Further increasing the truncation leads to more octahedronlike shapes. In the region $s \in(0.712,0.95]$, we find a Minkowski crystal [27], which melts into a stable pbcc phase before melting into a fluid. However, for $s \in$ $(0.95,1.0]$, we find that the intervening pbcc phase becomes metastable with respect to the Minkowski crystal-to-liquid phase transition (see also Ref. [31]), such that at $s=0.95$, an isotropic liquid-pbccMinkowski crystal triple point appears in the phase diagram. Typical configurations of plastic crystals and vacancy-rich simple-cubic phases can be found in the Supplemental Material [38]. The straight lines separating the phase boundaries for $s \in[0.374,0.712]$ at high packing fractions are a continuation of the subdivision that follows from the distinct crystal structures at close packing. Several simulations close to the boundaries (on either side) are performed, to show that within the numerical accuracy, there is no deviation from the vertical.

Now that we have described the phase diagram, let us return to the vacancy-rich sc phase for $s \in[0.00,0.374]$. We define the vacancy concentration $\alpha$ as the fraction of unoccupied sites in the sc crystal lattice. To determine the equilibrium vacancy concentration, we calculated the free energy (per particle per $\left.k_{B} T\right) f(\alpha)$ as a function of $\alpha$ for $s=0.05, s=0.15$, and $s=0.25$, at packing fraction $\phi=0.56$ using the method as described in Ref. [36]. Surprisingly, Fig. 3 shows that the minimum in $f(\alpha)$ shifts to higher $\alpha$ upon increasing the level of truncation $s$ at a fixed packing fraction. This is unlike the behavior observed for parallel cuboids (smooth-edged, parallel-aligned cubes) [37], which exhibit a constant vacancy concentration with increasing roundness at fixed $\phi$. The vacancies are delocalized along rows in the crystal lattice, in accordance with 


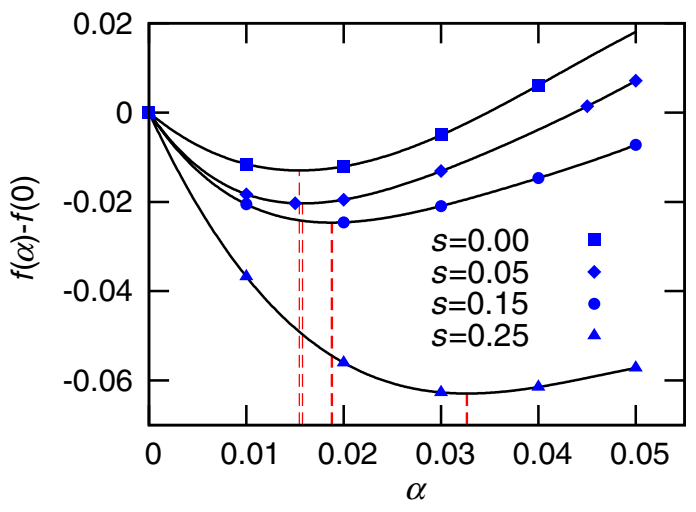

FIG. 3 (color online). Free-energy differences per particle $f=$ $F /\left(N k_{B} T\right)$ between a system with vacancies $f(\alpha)$ and the one without vacancies $f(0)$ as a function of the fraction of vacancies $\alpha$ for truncations $s=0, s=0.05, s=0.15$, and $s=0.25$ at packing fraction $\phi=0.56$. For $s=0$, the results are provided by the authors of Ref. [36]. The actual data points are plotted in thick blue symbols; the black line is a polynomial fit to the data. The dashed (red) vertical lines show the location of the minimum in the free energy, i.e., the equilibrium vacancy concentration $\alpha$.

the results of Ref. [36], since the particles can easily move with respect to each other and fill up the vacated space.

The free energy of a system with vacancy concentration $\alpha$ at a constant packing fraction $\phi$ and for a given truncation level $s$ is $f(\alpha)=f_{\text {comb }}(\alpha)+f_{\text {def }}(\alpha)$, where $f_{\text {comb }}(\alpha)$ accounts for the combinatorial entropy to place $n \equiv N-$ $N_{L}$ vacancies at $N_{L}$ lattice sites, and $f_{\text {def }}(\alpha)$ is the free energy of the crystal system that contains $n=\alpha N_{L}$ vacancies. In accordance with the observations of Ref. [36], we find that $f_{\text {def }}(\alpha)=f_{\text {def }}(0)+\alpha f_{1}$ represents the data accurately, suggesting weak interactions between vacancies. Here, $f_{\text {def }}(0)$ is the free energy of a system with no vacancies and $f_{1}>0$ is the free-energy cost to create a single vacancy. We observe that $f_{1}$ decreases with $s$ at fixed $\phi$, thereby explaining the increase in vacancy concentration with the level of truncation by the reduced free-energy cost to create a vacancy; see the Supplemental Material [38].

It is interesting to compare the present phase diagram to that of hard superballs [30,31] for which the shape interpolates also from cubes to octahedra, but via spheres instead of cuboctahedra. Although there are similarities between the two phase diagrams, such as stable plasticcrystal phases in the center of the phase diagram, there are also striking differences. (i) The stable plastic-crystal regimes are much smaller for polyhedral particles than for superballs. (ii) The phase boundaries of hard superballs change continuously as a function of the shape parameter, whereas the phase boundaries for truncated cubes exhibit sharp transitions. These observations lead to the idea that the more spread-out local curvature of the superballs tends to favor the formation of rotator phases and overall smoother phase behavior, whereas the polyhedral particles with flat faces and sharp edges prefer to align the flat faces to form crystals, which leads to sharp transitions even though $s$ varies smoothly.

Summarizing, we calculated the full phase diagram for a family of truncated cubes, which interpolates smoothly from a cube via a cuboctahedron to an octahedron. The phase diagram shows a remarkable diversity in crystal structures, despite the shape parameter changing smoothly. Of particular interest is the discovery of a fully degenerate crystal phase for certain levels of truncation $(s \approx 0.4)$, in which diagonally interlocked sheets of particles can move with respect to each other in only one direction. In addition, the latter system is remarkable since it exhibits a fluid state and three different bulk crystals upon increasing the pressure. Both of these qualities may make similarly shaped nanoparticles suitable for the creation of highly tunable functional materials, for which optical, electrical, and rheological properties vary strongly with the bulk pressure of the system. Finally, we showed that the equilibrium vacancy concentration, which is already unusually high for a system of cubes [36], increases even further by truncation at a given $\phi$ in contrast to the result for parallel cuboids [37].

Although it is tempting to define general guiding rules for the self-assembly of shape-anisotropic particles, the shape sensitivity revealed by the present study shows that one has to be cautious. After all, this also implies that the effect of experimentally inevitable size and shape polydispersity calls for further developments to analyze the stability of structures, e.g., along the lines of Refs. [44,45]. Our present results provide a solid basis for future studies of anisotropic particle systems and pave the way for a full understanding of the recent experimental studies performed on systems of nanoscopic truncated cubes.

We would like to thank F. Smallenburg and L. Filion for providing data for hard cubes and for useful discussions. M. D. and R. v. R. both acknowledge financial support by a NWO-Vici Grant.

*A.P. Gantapara@uu.nl

${ }^{\dagger}$ M. Dijkstra1@uu.nl

[1] Y. Sun and Y. Xia, Science 298, 2176 (2002).

[2] E. Matijevic, Acc. Chem. Res. 14, 22 (1981).

[3] X. Xia and Y. Xia, Nano Lett. 12, 6038 (2012).

[4] B. Wiley, T. Herricks, Y. Sun, and Y. Xia, Nano Lett. 4, 1733 (2004).

[5] Y. Wang, Y. Zheng, C. Z. Huang, and Y. Xia, J. Am. Chem. Soc. 135, 1941 (2013).

[6] J. Zeng, C. Zhu, J. Tao, M. Jin, H. Zhang, Z.-Y. Li, Y. Zhu, and Y. Xia, Angew. Chem., Int. Ed. Engl. 51, 2354 (2012).

[7] A. S. Barnard, X. M. Lin, and L. A. Curtiss, J. Phys. Chem. B 109, 24465 (2005).

[8] X. Zhang, C. Dong, J. Zapien, S. Ismathullakhan, Z. Kang, J. Jie, X. Zhang, J. Chang, C.-S. Lee, and S.-T. Lee, Angew. Chem., Int. Ed. Engl. 48, 9121 (2009). 
[9] H.-L. Wu, C.-H. Kuo, and M.H. Huang, Langmuir 26, 12307 (2010).

[10] W. H. Evers, B. Goris, S. Bals, M. Casavola, J. de Graaf, R. v. Roij, M. Dijkstra, and D. Vanmaekelbergh, Nano Lett. 13, 2317 (2013).

[11] H. R. Vutukuri, Ph.D. thesis, Utrecht University, 2012.

[12] Y. Ma, W. Li, E. C. Cho, Z. Li, T. Yu, J. Zeng, Z. Xie, and Y. Xia, ACS Nano 4, 6725 (2010).

[13] L. Rossi, S. Sacanna, W. T. M. Irvine, P. M. Chaikin, D. J. Pine, and A. P. Philipse, Soft Matter 7, 4139 (2011).

[14] S.C. Glotzer and M. J. Solomon, Nat. Mater. 6, 557 (2007).

[15] J. Henzie, M. Grünwald, A. Widmer-Cooper, P. L. Geissler, and P. Yang, Nat. Mater. 11, 131 (2012).

[16] Y. Bai, T. Yang, Q. Gu, G. Cheng, and R. Zheng, Powder Technol. 227, 35 (2012).

[17] J. Zhang, Z. Luo, B. Martens, Z. Quan, A. Kumbhar, N. Porter, Y. Wang, D.-M. Smilgies, and J. Fang, J. Am. Chem. Soc. 134, 14043 (2012).

[18] M. Eguchi, D. Mitsui, H.-L. Wu, R. Sato, and T. Teranishi, Langmuir 28, 9021 (2012).

[19] Z. Quan, W. S. Loc, C. Lin, Z. Luo, K. Yang, Y. Wang, H. Wang, Z. Wang, and J. Fang, Nano Lett. 12, 4409 (2012).

[20] J. J. Choi, K. Bian, W. J. Baumgardner, D.-M. Smilgies, and T. Hanrath, Nano Lett. 12, 4791 (2012).

[21] Y. Zhang, F. Lu, D. van der Lelie, and O. Gang, Phys. Rev. Lett. 107, 135701 (2011).

[22] L. Rossi, S. Sacanna, W. T. M. Irvine, P. M. Chaikin, D. J. Pine, and A. P. Philipse, Soft Matter 7, 4139 (2011).

[23] S. Torquato and Y. Jiao, Nature (London) 460, 876 (2009).

[24] J. de Graaf, R. van Roij, and M. Dijkstra, Phys. Rev. Lett. 107, 155501 (2011).

[25] U. Betke and M. Henk, Comput. Geom. 16, 157 (2000).

[26] C. B. Murray, C. R. Kagan, and M. G. Bawendi, Annu. Rev. Mater. Sci. 30, 545 (2000).

[27] H. Minkowski, Nachr. K. Ges. Wiss. Göttingen 11, 311 (1904).
[28] J. Burnet, Greek Philosophy, Part 1: Thales to Plato (Macmillan, London, 1914), 1st ed.

[29] J. V. Field, Arch. Hist. Exact Sci. 50, 241 (1997).

[30] R. D. Batten, F. H. Stillinger, and S. Torquato, Phys. Rev. E 81, 061105 (2010).

[31] R. Ni, A.P. Gantapara, J. de Graaf, R. van Roij, and M. Dijkstra, Soft Matter 8, 8826 (2012).

[32] P. F. Damasceno, M. Engel, and S. C. Glotzer, ACS Nano 6, 609 (2012).

[33] U. Agarwal and F. A. Escobedo, Nat. Mater. 10, 230 (2011).

[34] P.F. Damasceno, M. Engel, and S. C. Glotzer, Science 337, 453 (2012).

[35] G. van Anders, N. K. Ahmed, R. Smith, M. Engel, and S. C. Glotzer, arXiv:1304.7545.

[36] F. Smallenburg, L. Filion, M. Marechal, and M. Dijkstra, Proc. Natl. Acad. Sci. U.S.A. 109, 17886 (2012).

[37] M. Marechal, U. Zimmermann, and H. Löwen, J. Chem. Phys. 136, 144506 (2012).

[38] See Supplemental Material at http://link.aps.org/ supplemental/10.1103/PhysRevLett.111.015501 for more details regarding the simulation methods, free-energy calculations, and crystal structures.

[39] L. Filion, M. Marechal, B. van Oorschot, D. Pelt, F. Smallenburg, and M. Dijkstra, Phys. Rev. Lett. 103, 188302 (2009).

[40] A. Haji-Akbari, M. Engel, A. S. Keys, X. Zheng, R. G. Petschek, P. Palffy-Muhoray, and S.C. Glotzer, Nature (London) 462, 773 (2009).

[41] E. R. Chen, M. Engel, and S. C. Glotzer, Discrete Comput. Geom. 44, 253 (2010).

[42] J. de Graaf, L. Filion, M. Marechal, R. Roij, and M. Dijkstra, J. Chem. Phys. 137, 214101 (2012).

[43] D. Eberly, Intersection of Convex Objects: The Method of Separating Axes, http://www.geometrictools.com/.

[44] Y. Kallus and V. Elser, Phys. Rev. E 83, 036703 (2011).

[45] U. Agarwal and F. A. Escobedo, J. Chem. Phys. 137, 024905 (2012). 


\title{
Supplemental Material \\ Phase Diagram and Structural Diversity of a Family of Truncated Cubes: Degenerate Close-Packed Structures and Vacancy-Rich States
}

\author{
Anjan P. Gantapara, ${ }^{1, *}$ Joost de Graaf, ${ }^{1}$ René van Roij, ${ }^{2}$ and Marjolein Dijkstra ${ }^{1, \dagger}$ \\ ${ }^{1}$ Soft Condensed Matter, Debye Institute for Nanomaterials Science, \\ Utrecht University, Princetonplein 5, 3584 CC Utrecht, The Netherlands \\ ${ }^{2}$ Institute for Theoretical Physics, Utrecht University, \\ Leuvenlaan 4, 3584 CE Utrecht, The Netherlands
}

(Dated: June 14, 2013)

\section{DEFINITION OF THE FAMILY OF TRUNCATED CUBES}

We define our family of truncated cubes using a simple mathematical expression for the location of the vertices. The line segments that connect these vertices can only be assigned in one (unique) way to obtain a truncated cube. The vertices of a truncated cube may be written as a function of the shape parameter $s \in[0,1]$ :

$$
\{\mathbf{v}(s)\}=\left\{\begin{array}{c}
\left(1-\frac{4}{3} s^{3}\right)^{(-1 / 3)} \mathcal{P}_{D}\left( \pm\left(\frac{1}{2}-s\right), \pm \frac{1}{2}, \pm \frac{1}{2}\right)^{T} \\
s \in\left[0, \frac{1}{2}\right] \\
\left(\frac{4}{3}-4 \lambda^{3}\right)^{(-1 / 3)} \mathcal{P}_{D}( \pm(1-\lambda), \pm \lambda, 0)^{T} \\
\lambda \equiv 1-s \in\left[0, \frac{1}{2}\right]
\end{array},\right.
$$

where $\mathcal{P}_{D}$ is a permutation operation that generates all permutations of each element in the sets of 8 and 4 vertices spanned by the \pm -operations, respectively. All duplicate vertices are removed after letting $\mathcal{P}_{D}$ act. The ' $T$ ' indicates transposition. The prefactors ensure that the truncated cubes are normalized to unit volume. As mentioned in the main text, several Platonic and Archimedean solids are members of this family: $s=0$ a cube, $s=(2-\sqrt{2}) / 2 \approx 0.292893$ a truncated cube, $s=1 / 2$ a cuboctahedron, $s=2 / 3$ a truncated octahedron, and $s=1$ an octahedron; these are depicted in Fig. 1a.

\section{METHODS}

\section{A. Determining the Close-Packed Structures}

The simulations by which the close-packed structures were derived, are based on the floppy-box Monte Carlo (FBMC) method $[1,2]$ in combination with the separating-axis-based overlap algorithm [3]. We obtained

\footnotetext{
*Electronic address: A.P.Gantapara@uu.nl

†Electronic address: M.Dijkstra1@uu.nl
}

the densest crystal structure and the corresponding packing fraction $\phi$ as a function of the level of particle truncation $s$ by considering 1,000 equidistant points in $s \in[0,1]$. For each point we prepared systems of truncated particles in a dilute phase, typically with packing fraction $\phi \approx 0.001$. We increased the reduced pressure in 100 steps according to a geometric series from $p=1$ to $p \approx 10^{5}$ over $4 \cdot 10^{6}$ Monte Carlo (MC) cycles in order to compress these systems to a high-density crystalline state. This pressure increase was typically applied a total of 1,000 times for $N=1$ particles in the unit cell and for each shape. We restricted ourselves to $N=1$ particles in the unit cell, because the truncated cubes are all centrosymmetric. We only considered $N=2, \ldots, 6$ for 14 conveniently chosen values of $s$, located in the center of the regions indicated in Fig. 1, as will be justified shortly. For these $N>1$ systems we obtained roughly the same value of $\phi$ and also the same crystal structures. The densest crystal-structure candidate was selected and allowed to compress further for another $10^{6} \mathrm{MC}$ cycles at $p=10^{6}$ to achieve 5 decimals of precision in $\phi$. In practice, these final cycles of compression did not improve the packing fraction substantially. Figure 1b shows $\phi$ as a function of $s$. Note that the packing fraction 'curve' is continuous, but has discontinuities in its first derivative. To double check our result, we considered another set of FBMC runs. We used several of the 1,000 denselypacked crystals as our initial configuration and we varied $s$ around these points at high pressure to study the evolution of their structure. Steps of $10^{-5}$ in $s$ were used and for each step the system is expanded to remove any overlaps, before re-compressing it at $p \approx 10^{5}$. The packing fractions we obtained showed good correspondence with our original result, but this correspondence failed for a transition between two crystal structures. The consecutive method would often become stuck in the lower density structure that corresponded to the morphology of the crystal phase it came from.

The unit cell for $N=1$ truncated cubes can be specified by three vectors $\mathbf{v}_{i}(i=1,2,3)$ that are implicitly $s$ dependent. The structure spanned by these three vectors can also be described by the length $v_{i}=\left|\mathbf{v}_{i}\right|$ of the vectors and the angles $\theta_{i j}(i<j=1,2,3)$ between them. Note that we ignored the orientation of the particle with respect to the unit cell here. In order to give an unbiased comparison of the different vectors we used lattice 
(a)
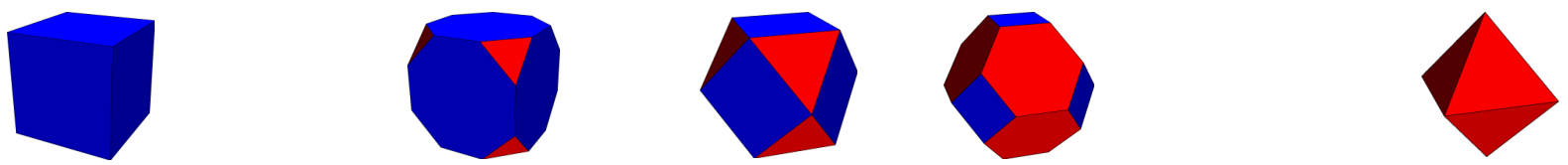

(b)

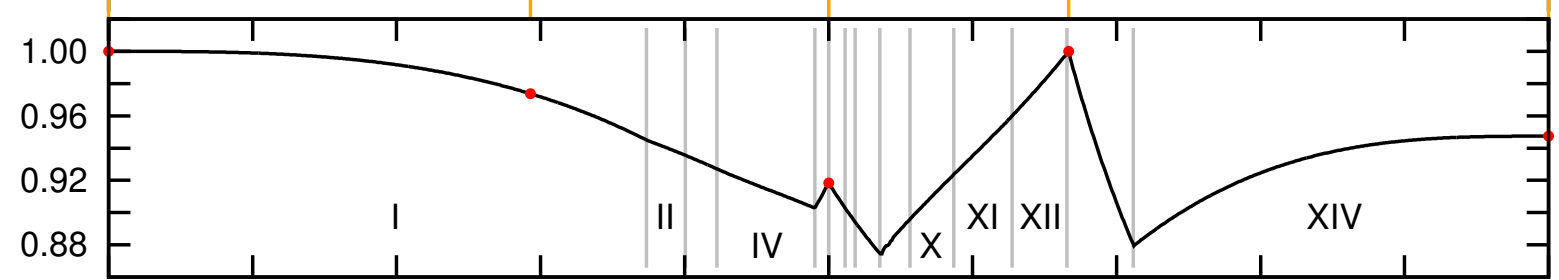

(c)

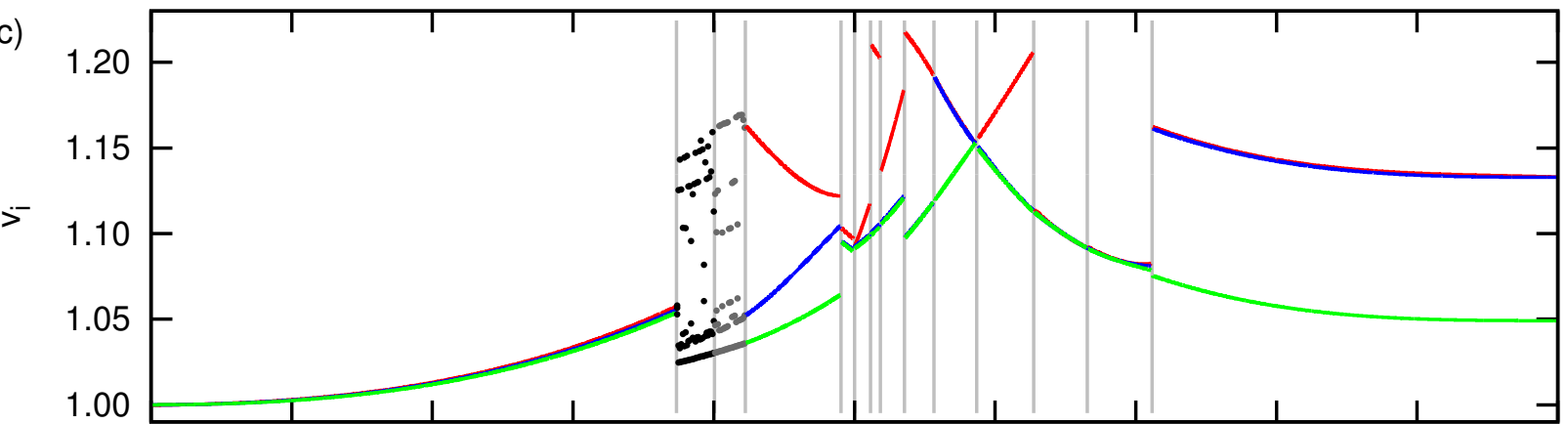

(d)

Figure 1: (a) Five examples of truncated cubes (Platonic and Archimedean solids only) for levels of truncation $s$ corresponding to the orange lines: $s=1$ a cube, $s=(2-\sqrt{2}) / 2 \approx 0.293$ a truncated cube, $s=1 / 2$ a cuboctahedron, $s=2 / 3$ a truncated octahedron, and $s=1$ an octahedron. (b) The packing fraction $\phi$ for the close-packed structures as a function of $s$. The values for the five solids shown in (a) are given by red dots. (c) The length $v_{i}(i=1,2$, and 3$)$ of the three lattice vectors, indicated in red, green, and blue, that span the unit cell of the densest crystal structure as a function of $s$. Not every line is clearly visible, since there is some overlap. In the region where the black and gray dots are used $(s \in[0.37,0.40]$ and $s \in[0.40,0.42])$, there appears to be a degeneracy in the crystal structures, as is explained in the text. (d) The cosine of the angles $\theta_{i j}(i<j=1$, 2 , and 3) between the three vectors that span the unit cell as a function of $s$. Gray vertical lines partition the $s$-domain into 14 pieces with a 'different' crystal structure, based on the discontinuities shown in the $v_{i}$ and $\cos \theta_{i j}$ results. These regions are numbered with roman numerals in (b); only those regions large enough to accommodate a label are numbered, but the numbering can be continued from left to right, in the unnumbered regions.

reduction [4] to ensure that for each unit cell the surface to volume ratio is minimal. These numerical results are shown in Fig. 1(c,d). By analyzing the $v_{i}$ and $\theta_{i j}$, as well as the location of the kinks in the $\phi$-curve, we were able to partition the $s \in[0,1]$ domain into 14 distinct regions. This is the reason behind our choice of 14 verification points for $N>1$ simulations. For a discussion of the crystal structures in the different regions, we refer the reader to Sec. IV. 


\section{B. Equations of State and Mesophase Structures}

We used the close-packed crystal structures obtained from the FBMC calculations as initial configurations for variable-box-shape isothermal-isobaric (NPT) Monte Carlo simulations, to study the phase behavior at intermediate pressures. Initial configurations of 300 to 600 particles were prepared and melted to determine the equations of state (EOSs) for the various phases. Typical equilibration and production times were around $1.2 \cdot 10^{6}$ and $2 \cdot 10^{6}$ Monte Carlo Sweeps (MCS), respectively. One MCS is defined as $N$ Monte Carlo trial moves (translation, rotation, volume change, or deformation of the box, respectively), where $N$ is the number of particles in the system. We sampled the lattice vectors, as well as the average positions and orientations of the particles as a function of packing fraction and truncation parameter $s$. The sampling was done on an interval of 100 MCS to avoid correlated configurations. Using these results we set up regular NPT simulations (possibly with a triclinic box shape) to more accurately sample the EOSs for all phases with larger system sizes of 1,000 to 2,000 particles, including the liquid phase. In addition, we employed these results to set up the Einstein crystal [5] for the free-energy calculations.

Several typical configurations of crystalline phases slightly above fluid coexistence are displayed in Fig. 2 to give an impression of the mesophases that occur in the systems we studied. We have also added a movie file to the supplemental information 'pbct.mpg', which shows the plastic-body-centered tetragonal (pbct) phase for truncation $s=0.546$ and pressure $p^{*}=11.0$, to illustrate that a bct structure can be present for particles that rotate. On the top-left corner the entire simulation box is shown for an NPT simulation containing 512 particles. An enlarged pbct unit cell is shown from the front (topright corner), from the bottom (bottom-left corner), and from the side (bottom-right corner); with all the neighboring particles hidden. The change in the color of the particles is related to the orientation of the particles, the more the particle is aligned with the lattice vectors of the Einstein crystal, the more bright green the color is; the more the particle is misaligned, the more it tends to a blue color.

\section{Free-Energy Calculations and Confining Potentials}

We obtained the dimensionless free energy per particle $f=\beta F / N$ as a function of packing fraction $\phi$ (or equivalently the density $\rho$ ) for the entire density range by thermodynamic integration [6] over the EOS, from reference density $\rho_{0}$ to the density of interest $\rho$ :

$$
f(\rho)=f\left(\rho_{0}\right)+\int_{\rho_{0}}^{\rho} \frac{\beta P\left(\rho^{\prime}\right)}{\rho^{\prime 2}} \mathrm{~d} \rho^{\prime} .
$$

Here $f\left(\rho_{0}\right) \equiv \beta \mu\left(\rho_{0}\right)-\beta P\left(\rho_{0}\right) / \rho_{0}$ is the reduced Helmholtz free energy per particle at density $\rho_{0}$, with $\beta=1 / k_{\mathrm{B}} T, T$ the temperature and $k_{\mathrm{B}}$ Boltzmann's constant, $\mu\left(\rho_{0}\right)$ the chemical potential, and $P\left(\rho_{0}\right)$ the pressure. The Helmholtz free energy at reference density $\rho_{0}$ was obtained as follows.

- In the fluid phase we used Widom's particle insertion method [7] to obtain the free energy. This method was employed at relatively low densities to obtain small error bars. We performed the calculations at $\phi \approx 0.2$. We note that there were no finite size effects within the computational accuracy for the particle insertion method.

- In the crystal phase we used the Einstein integration method $[6,8,9]$. The reduced Helmholtz free energy per particle $f=\beta F / N$ of a crystal is given by:

$$
\begin{aligned}
f(\rho)= & f_{\text {Einst }}\left(\lambda_{\max }\right)- \\
& \frac{1}{N} \int_{0}^{\lambda_{\max }} \mathrm{d} \lambda\left\langle\frac{\partial \beta U_{\text {Einst }}(\lambda)}{\partial \lambda}\right\rangle,
\end{aligned}
$$

where $f_{\text {Einst }}$ denotes the reduced free energy per particle of the ideal Einstein crystal, which is given by:

$$
\begin{aligned}
f_{\text {Einst }}\left(\lambda_{\max }\right)= & -\frac{3(N-1)}{2 N} \log \left(\frac{\pi}{\lambda_{\max }}\right)+ \\
& \log \left(\frac{\Lambda_{t}^{3} \Lambda_{r}}{v_{p}}\right)+\frac{1}{N} \log \left(\frac{v_{p}}{V N^{1 / 2}}\right)- \\
& \frac{1}{N} \log \left\{\frac{1}{8 \pi^{2}} \int \mathrm{d} \theta \sin (\theta) \mathrm{d} \phi \mathrm{d} \chi \times\right. \\
& \left.\exp \left[-\frac{\lambda_{\max }}{k_{B} T}\left(\sin ^{2} \psi_{i a}+\sin ^{2} \psi_{i b}\right)\right]\right\},
\end{aligned}
$$

with $v_{p}$ the volume of a particle. $U_{\text {Einst }}(\lambda)$ denotes the harmonic potential that fixes the particles to the respective Einstein lattice positions:

$$
\begin{array}{r}
\beta U_{\text {Einst }}(\lambda)= \\
\lambda \sum_{i=1}^{N}\left[\left(\mathbf{r}_{i}-\mathbf{r}_{i, 0}\right)^{2} / v_{p}^{2 / 3}+\left(\sin ^{2} \psi_{i a}+\sin ^{2} \psi_{i b}\right)\right],
\end{array}
$$

with $\left(\mathbf{r}_{i}-\mathbf{r}_{i, 0}\right)$ the displacement of particle $i$ from its position in the ideal Einstein crystal. The angles $\psi_{i a}$ and $\psi_{i b}$ are the minimum angles between vectors, $\mathbf{a}$ and $\mathbf{b}$, describing the orientations of the particles in the ideal Einstein crystal and the equivalent vectors that describe the orientation of the particle in the actual crystal, respectively. When $\lambda$ is large the particles translational and orientational displacements are frozen, while at lower $\lambda$ 's the particles freely displace and rotate. They thereby not only explore the possible states, but also the underlying degenaracy coming from the symmetry of 

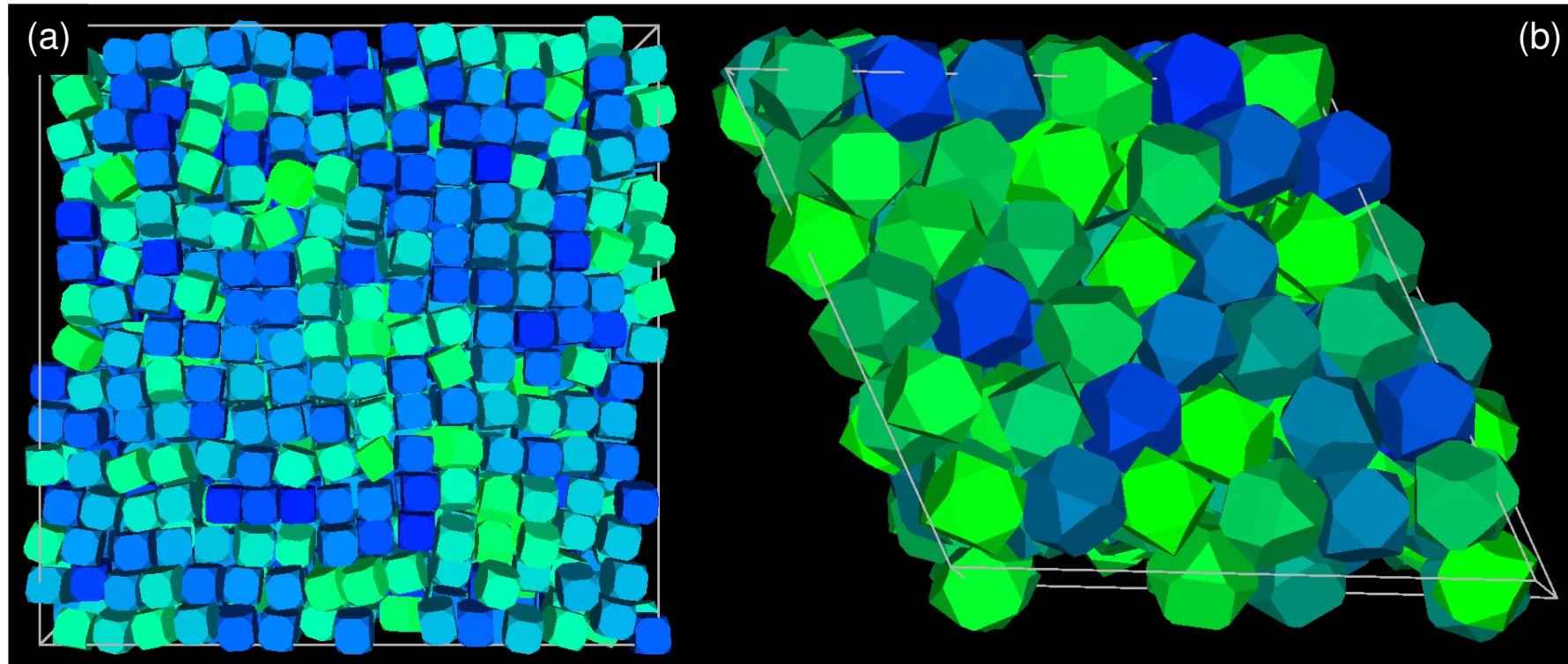

(c)

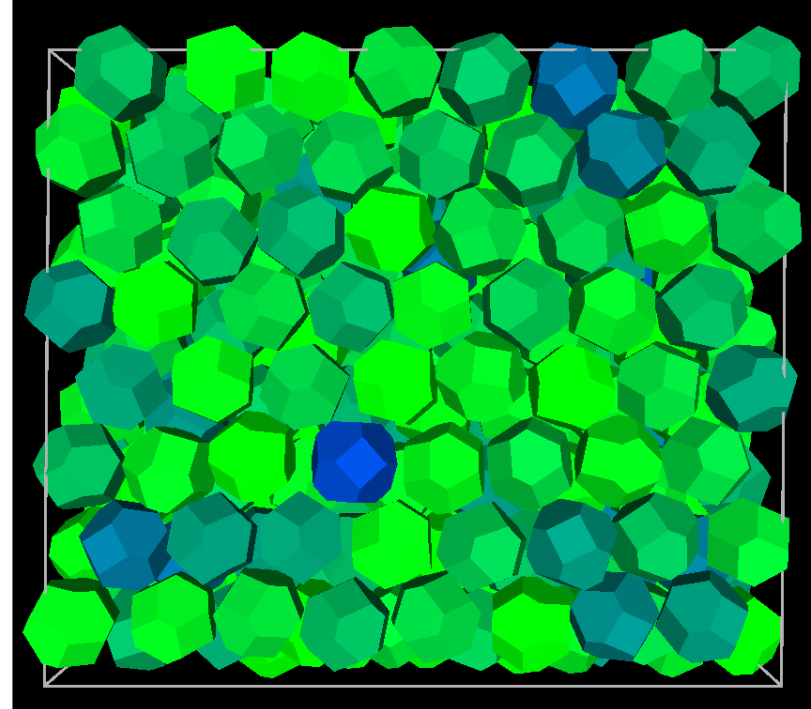

(d)

Figure 2: Several snapshots of our isothermal-isobaric $(N P T)$ simulations showing the various crystal structures that form in our family of truncated cubes. (a) Equilibrium vacancy-rich simple cubic crystal for a truncation of $s=0.25$ at packing fraction $\phi=0.56$. The simulation was performed for $N=3,235$ particles. In this system the vacancy concentration was found to be $\alpha=0.032$. (b) Plastic hexagonal-close-packed (PHCP) phase for $s=0.411$ and $\phi=0.6$ in a box containing $N=216$ particles. (c) Plastic body-centered-tetragonal (pbct) phase for $s=0.607$ and $\phi=0.58$ in a box containing $N=512$ particles. (c) Plastic body-centered-cubic (PBCC) phase for $s=0.900$ and $\phi=0.52$ in a box containing $N=250$ particles. The coloring used here indicates the level of alignment of these particles with the lattice vectors of the Einstein crystal, as explained in the text.

the particle itself. The translational and rotational thermal wavelengths $\Lambda_{t}$ and $\Lambda_{r}$ are set to 1 in our calculations. We mostly used the same system sizes of 700 to 1,500 particles to compute the free energies for the (plastic) crystal phases. We found that finite-size scaling (FSS) was only necessary in the octahedron regime, i.e., $s \approx 1$, to establish the phase diagram. For such high levels of truncation the free-energy differences between the various phases at coexistence proved to be very small, see Ref. [5]. For the other phase transitions the free energies obtained without FSS proved to be sufficient to accurately determine the phase boundaries.

- For the free-energy calculations of a plastic-crystal (rotator) phase, we followed the approach of the authors of Ref. [10], who introduced a method, which allows for a continuous transition from a non- 
interacting plastic-crystal to an interacting plasticcrystal phase of hard truncated cubes. We used a tunable soft-to-hard interaction potential between the particles

$$
\varphi(i, j)=\left\{\begin{array}{cl}
\gamma[1-A(1+\zeta(i, j))] & \text { if } \zeta(i, j)<0 \\
0 & \text { otherwise }
\end{array}\right.
$$

where $\zeta(i, j)$ is the overlap potential defined in Ref. [11], which is negative when two particles $i$ and $j$ overlap and positive otherwise, and $\gamma$ is the integration parameter, which runs from $\gamma=0$ (noninteracting) to $\gamma=\gamma_{\max }$, for which the system interacts fully. In our calculations we used $A=0.9$ following Ref. [12]. The dimensionless Helmholtz free energy per particle $f=\beta F / N$ of the plastic crystal is given by:

$$
\begin{aligned}
f(\rho)= & f_{\text {Einst }}\left(\lambda_{\max }\right)- \\
& \frac{1}{N} \int_{0}^{\lambda_{\max }} \mathrm{d} \lambda\left\langle\frac{\partial \beta U_{\text {Einst }}(\lambda)}{\partial \lambda}\right\rangle_{\gamma_{\max }}+ \\
& \frac{1}{N} \int_{0}^{\gamma_{\max }} \mathrm{d} \gamma\left\langle\frac{\partial \sum_{i \neq j}^{N} \beta \varphi(i, j)}{\partial \gamma}\right\rangle_{\lambda_{\max }} .
\end{aligned}
$$

- We calculated the free energy of a solid with a fixed vacancy concentration $\alpha=\left(N_{L}-N\right) / N_{L}$, with $N$ the number of particles and $N_{L}$ the number of lattice sites, according to the procedures outlined in Ref. [13]. The dimensionless free energy per particle $f_{\text {Einst }}^{\mathrm{vac}}$ of a non-interacting Einstein crystal with vacancies is given by:

$$
f_{\text {Einst }}^{\mathrm{vac}}\left(\lambda_{\max }\right)=f_{\text {Einst }}\left(\lambda_{\max }\right)+f_{\text {comb }},
$$

where $f_{\text {comb }}$ is the combinatorial entropy associated with placing $N$ particles on $N_{L}$ lattice sites:

$$
f_{\mathrm{comb}}=-\frac{1}{N} \log \left(\frac{N_{L} !}{N !\left(N_{L}-N\right) !}\right) .
$$

For more details about the implementation of the free-energy calculations, we refer to the methods section of Ref. [13]. We used $N_{L}=15^{3}=3,375$ for the free-energy calculation at $\phi=0.56$.

\section{FREE ENERGY OF VACANCIES}

We calculated the free energies for a simple cubic (sc) crystal phase of truncated hard cubes with a shape parameter $s=0.0,0.05,0.15$, and 0.25 and packing fraction $\phi=0.56$ as a function of the vacancy concentration $\alpha=\left(N_{L}-N\right) / N_{L}$ as described in Sec. II C. In Fig. 3, we present the dimensionless free energy per particle $f_{\text {def }}$ as a function of $\alpha$ without taking into account the combinatorial entropy, i.e., $f_{\text {def }}(\alpha)=f(\alpha)-f_{\text {comb }}(\alpha)$. In accordance with the observations of Ref. [13], $f_{\text {def }} v s$.

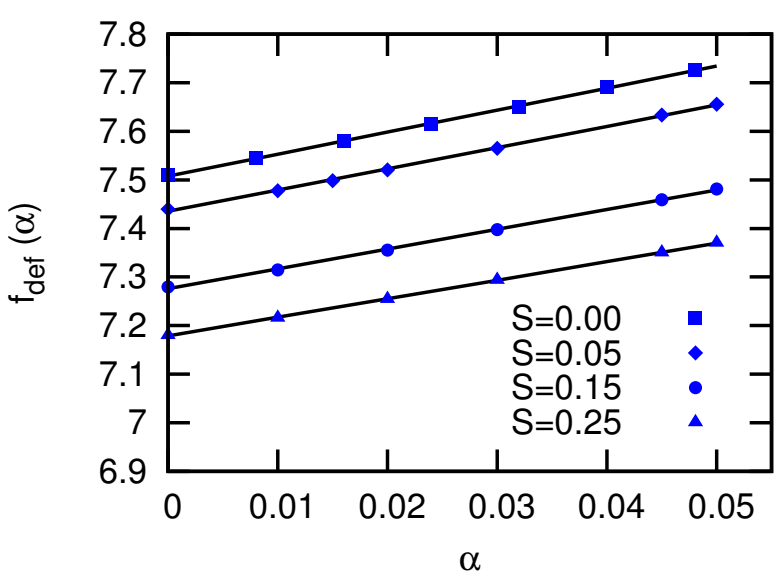

Figure 3: The reduced free energy per particle $f_{\text {def }}(\alpha)$ as a function of vacancy concentration $\alpha$ for a simple cubic crystal phase of truncated cubes with shape parameter $s=0,0.05$, 0.15 , and 0.25 and packing fraction $\phi=0.56 . f_{\text {def }}(\alpha)$ does not account for the combinatorial entropy, i.e., $f_{\text {def }}(\alpha)=f(\alpha)-$ $f_{\text {comb }}(\alpha)$.

Table I: The dimensionless free energy per particle for a simple cubic crystal of truncated cubes with shape parameter $s=0$, $0.05,0.15$, and 0.25 and packing fraction $\phi=0.56$. From left to right we specify the truncation parameter $s$, the free energy for a system with zero vacancies $f(0)$ and the free energy cost to create a single vacancy $f_{1}$ (without the combinatorial contribution) as obtained by Monte Carlo simulations.

\begin{tabular}{|c|c|c|}
\hline \hline$s$ & $f(0)$ & $f_{1}$ \\
\hline 0.00 & 7.5077 & 4.52640 \\
0.05 & 7.4354 & 4.36892 \\
0.15 & 7.2762 & 4.06024 \\
0.25 & 7.1788 & 3.81633 \\
\hline
\end{tabular}

$\alpha$ in Fig. 3 shows a linear behavior suggesting that the vacancies are only weakly interacting. Assuming the vacancies to be non-interacting, as explained in the main text, one can write the free energy of a crystal phase as

$$
f_{\text {def }}(\alpha)=f(0)+\alpha f_{1}
$$

where $f(0)$ is the dimensionless free energy per particle of a crystal with no vacancies and $f_{1}>0$ is the reduced free energy cost to create a single vacancy (without the combinatorial component). Fitting the results as displayed in Fig. 3 with Eq. 8, we determined $f_{1}$ for truncated cubes as a function of shape parameter $s$. The results are tabulated in Table I. Surprisingly, we found that the free energy cost to create a vacancy $f_{1}$ decreases with increasing $s$ at packing fraction $\phi=0.56$. Hence, the equilibrium vacancy concentration increases with particle truncation $s$, also see the main text.

In the phase diagram of truncated cubes, shown in Fig. 2 of the manuscript, we did not take into account the shift in the coexistence densities effected by the high vacancy concentrations in the sc phase. Smallenburg et al. have 
shown that the coexistence densities for the phase diagram change by only a few percent if the vacancies are taken into account for the high-vacancy phases [13]. We therefore feel justified in this omission, since the influence in the vacancies leads to a minute change in the location of the phase boundaries for the sc-fluid coexistence densities. There is no indication that the other phases suffered from such high vacancy concentrations and any phase boundaries determined for these coexistences can therefore be taken as precise within the numerical uncertainty.

\section{DENSE PACKINGS AND CRYSTAL STRUCTURES}

In this section we discuss the properties of the closepacked structures that are obtained using the methods described in Sec. II A. Figure 5 shows the crystal structure in the center of each of the 14 regions that we found in Fig. 1. There is a strong difference between the domains $s<1 / 2$ and $s>1 / 2$. Geometrically the cuboctahedron $(s=1 / 2)$ is the transition point between shapes which have a more cube-like nature and shapes which have a more octahedron-like nature. It is therefore not surprising that the crystal structures in the two regions $(s<1 / 2$ and $s>1 / 2)$ appear to have a deformed simple cubic (dsc) symmetry and a deformed body-centered tetragonal (dbct) symmetry, respectively. We illustrate this in Fig. 5 where we show the most orthorhombic unit cell: $N=1$ for $s<1 / 2$ and $N=2$ for $s>1 / 2$. A remarkable result is the stability of the Minkowski crystal [14], which is the densest-packed Bravais-lattice structure for octahedra [15], under variations in $s$. For all $s \in[0.71,1]$, we find a Minkowski crystal in the densepacked limit, which can be inferred from the horizontal $\cos \theta_{i j}$ lines in Fig. 1d. The scaled length of the vectors $v_{i} \phi^{-1 / 3}$ is also constant on this domain.

Let us now examine the crystal structures in the 14 regions identified by the discontinuities in the vectors of the unit cell. In literature it has become commonplace to assign atomic equivalents to structures observed in simulations or experiments. For example, this is done for binary mixtures of spheres $[1,16]$, a family of truncated tetrahedra [17], several faceted particles [18], and systems of nanoparticles $[19,20]$. We attempted to follow suit by determining the symmetry group of the structures in Fig. 5 using FindSym [21] and by subsequently assigning an atomic equivalent [22]. However, we found that a description in terms of atomic equivalents inadequately captures the richness in crystal structure, since particle orientation is not taken into account. Moreover, for many of our structures we are unable to determine a nontrivial space group using FindSym. We therefore resorted to visual analysis and we used this to group the 14 regions in Fig. 1 based on similarities between the respective structures.

- I In this region $(s \in[0.00,0.37])$ we obtained a con-

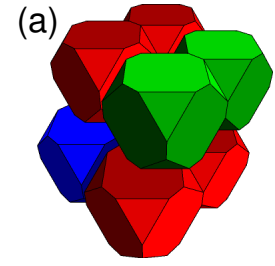

(c)

(b)

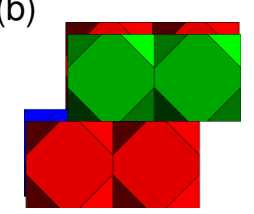

(d)

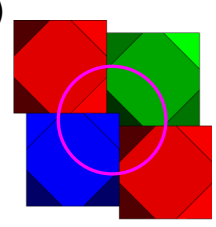

(e)
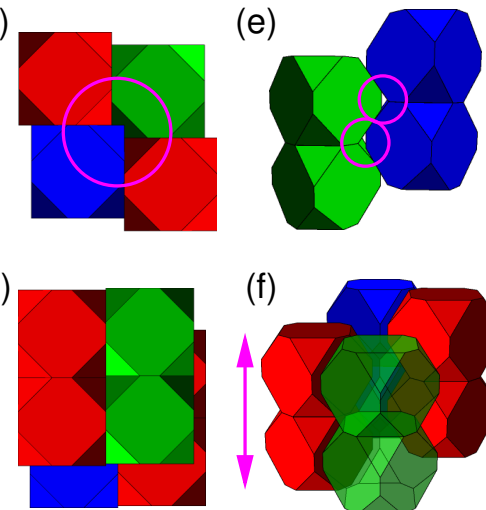

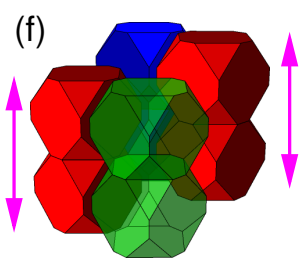

Figure 4: A visual representation of the degenerate crystal structure; we consider the value $s=0.387$ in this figure. Pairs of truncated cubes, for which the octahedral faces are aligned (columns), are color-coded. Different viewpoints are displayed for a piece of crystal consisting of 8 particles: (a) Bird's eye view, (b) side view, (c), front view, and (d) top view of this structure. In (c) we use a magenta circle to indicate that the blue column is interlocking with the green column in a diagonal way. (e) A diagonal view of the crystal structure, where the red columns have been removed. Magenta circles show the interlocking. (f) The two red columns are not interlocking with the blue and green column, allowing for freedom of motion in the direction of the magenta arrows. The green column is made translucent to better illustrate the properties of this crystal structure.

tinuous and uniform distortion of the simple cubic structure for cubes. For $s=0$ the particles form a sc crystal, which has the same morphology as $\alpha \mathrm{Po}$ ( $\alpha$-Polonium) [22]. The uniformly deformed simple cubic (udsc) structure we found for $s>0$ is similar to that of $\beta \mathrm{Po}_{0}[22]$. We verified this deformed quality for values as low as $s=10^{-5}$.

- II \& III For these two regions $(s \in[0.37,0.40]$ and $s \in[0.40,0.42])$ we found that there is a degeneracy in the crystal-structure candidates that achieve the densest-known packing. Although certain structures appear favored over others, there is no clear relation between the structure and $s$. However, the packing fraction $\phi$ of the close-packed crystals is continuous in these regions. The observed degeneracy can be explained by the formation of sheets consisting of diagonally-interlocked columns, which can slide up or down (in the direction of the columns) with respect to each other, as shown in Fig. 4. For $s=0.387$, the truncated cubes are arranged in a dsc crystal lattice, where the particles form columns that are interlocked in a diagonal way. These structures are referred to as mono-interlocking distorted simple cubic (MI dsc) crystals. This diagonal interlocking together with the close-packing condition prevents lateral motion in the plane normal to the column's direc- 


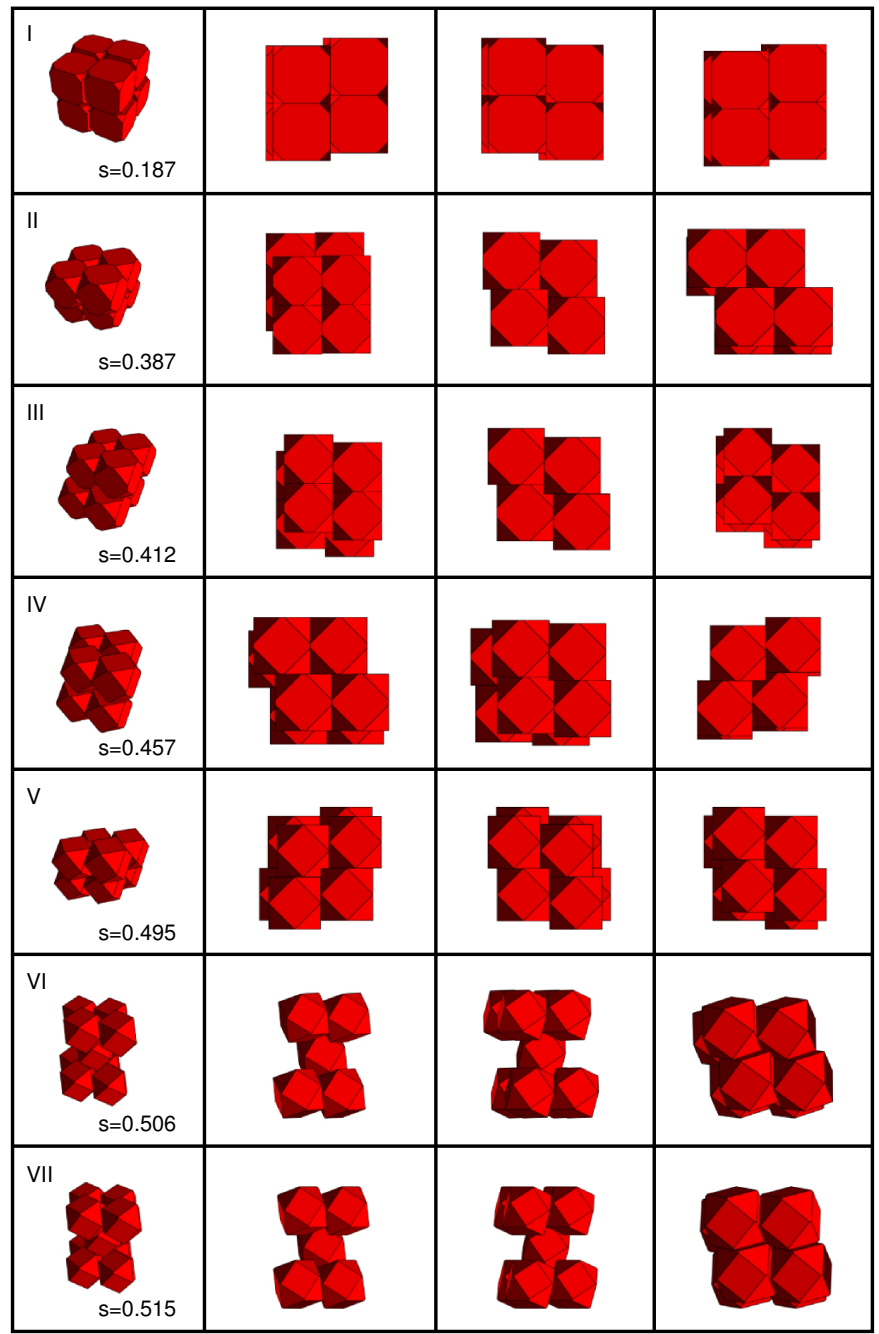

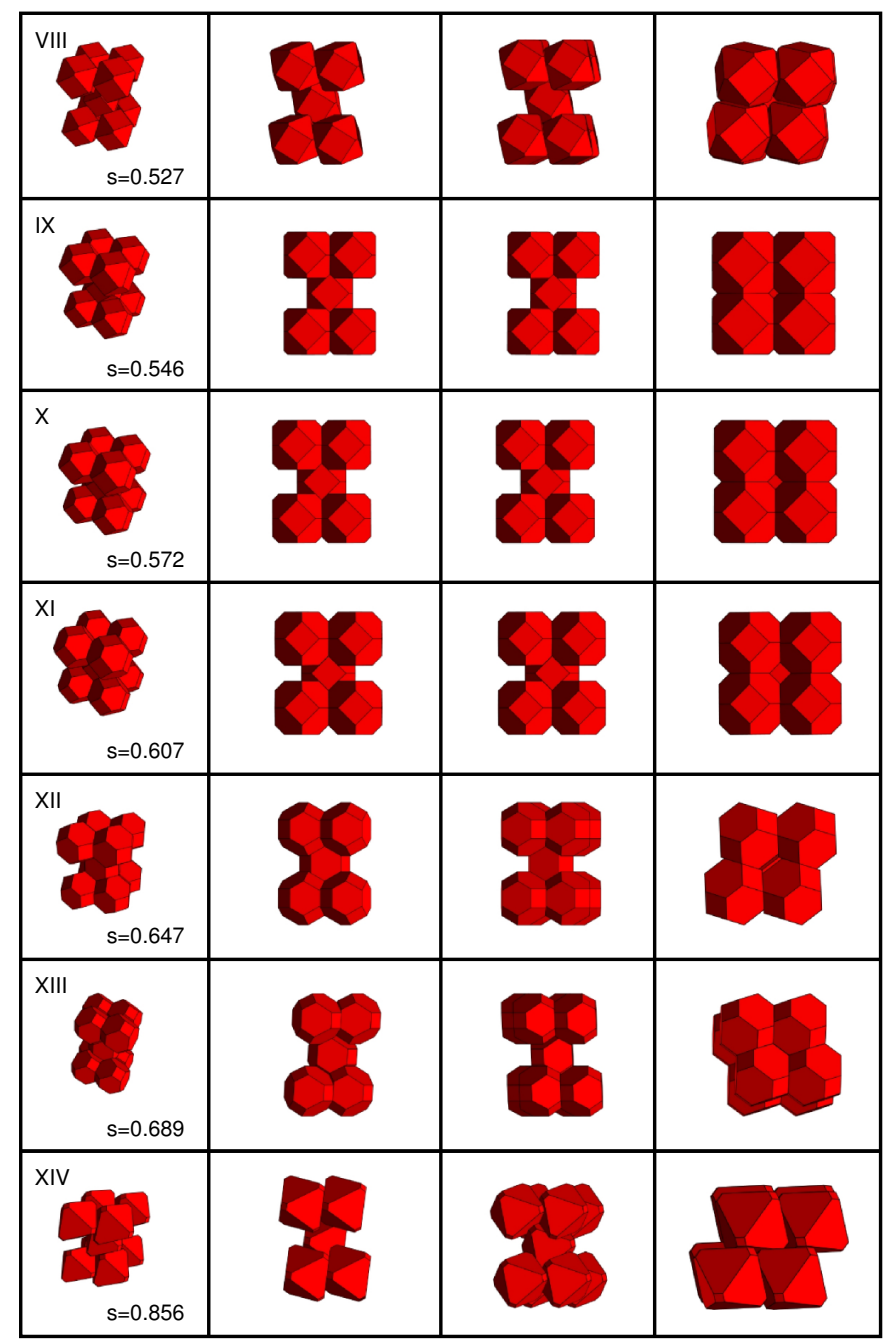

Figure 5: Visual representations of the crystal structures obtained for the first 7 regions (left) and the last 7 regions (right) of Fig. 1. From left to right each entry (row) contains a bird's eye view, the front view, the side view, and the top view of this structure. The Roman numeral in the top-left corner gives the relevant domain in Fig. 1. The truncation parameter $s$ for these structures is given in the bottom-right corner.

tion. However, since the system is not fully interlocked, motion in the direction of the columns is possible for the diagonally interlocked sheets. We refer to these structures as mono-interlocking distorted simple cubic (MI dsc) crystals. This degeneracy is different from the degeneracy that occurs in structures consisting of cubes or hexagonal prisms for instance, since such systems allow lateral freedom of movement of columns or (perpendicular to the columns) of sheets of aligned particles. That is, there is possible freedom of motion in three directions, albeit not necessarily at the same time. The interlocking nature of the MI dsc phase only allows for movement in one direction only, namely parallel to the columns, which may lead to strong rheological differences between this structure and, e.g., the sc structure for cubes.
- IV For this region $(s \in[0.42,0.49])$ we find a dsc phase that is interlocking in two directions: a biinterlocking dsc (BI dsc) phase. For each instance of interlocking two degrees of translational motion are frozen out. This implies that the BI dsc structure is completely fixed, which is confirmed by the unicity of the $v_{i}$ and $\theta_{i j}$ results in Fig. $1(\mathrm{c}, \mathrm{d})$.

- V In this region $(s \in[0.49,0.50])$ we observed a tri-interlocking dsc (TI dsc) phase.

- VI - VIII Here $(s \in[0.50,0.51],[0.51,0.52]$, and $[0.52,0.54])$ we found structures that are best described by a distorted body-centered tetragonal (dbct) structure. The truncated cubes in these crystals are not aligned with the axes of the unit cell. It is unclear to what extent structures in regions VI, VII, and VIII are the same. The smooth 
flow of the $\phi$-curve (Fig. 1b), as well as their appearance, $\mathrm{s}$ implies continuity, but the jumps in the values of $v_{i}$ and $\theta_{i j}$ [Fig. $\left.1(\mathrm{c}, \mathrm{d})\right]$ suggest otherwise.

- IX - XI These structures $(s \in[0.54,0.56]$, $[0.56,0.59]$, and $[0.59,0.63])$ have a body-centered tetragonal (bct) morphology, for which the particles are aligned with the lattice vectors of the unit cell. It is surprising that the structures in region XII exhibit a dbct morphology, since regions IX XII share the same smooth piece of $\phi$-curve, see Fig. 1b. This leads us to conclude that a smooth dependence of $\phi$ on $s$ is not indicative of uniformity in crystal structure. The strong similarity between the crystal structures in regions IX - XI and the apparent smooth transition between structures from region IX to $\mathrm{X}$ and from $\mathrm{X}$ to $\mathrm{XI}$, also leads us to conclude that discontinuities in the properties of unit cell are not indicative of discontinuities in the properties of the crystal structure.

- XII \& XIII These two dbct structures $(s \in$
$[0.63,0.67]$ and $s \in[0.67,0.71])$ are different from the dbct structures in regions VI - VIII, since the particles appear to be aligned with the lattice vectors of the unit cell. Moreover, crystals in region XIII are unusual, since there are large 'voids' in the structure. That is, for all other structures we found that the largest facets of a particle are always in contact with a facet of another particle. This is not the case in region XIII, because there is a substantial gap between some of the hexagonal facets.

- XIV The Minkowski crystal of region XIV $(s \in$ $[0.71,1.00])$ is also peculiar. It is the only structure which does not undergo some form of reorganization upon varying the level of truncation. It is worthwhile to study the origin of this apparent stability, which sharply contrasts with the immediate distortion found around $s=0$. However, this goes beyond the scope of the current investigation.
[1] Filion, L.; Marechal, M.; van Oorschot, B.; Pelt, D.; Smallenburg, F.; Dijkstra, M. Phys. Rev. Lett. 2009, 103, 188302.

[2] de Graaf, J.; Filion, L.; Marechal, M.; Roij, R.; Dijkstra, M. J. Chem. Phys. 2012, 137, 214101.

[3] Eberly, D. Intersection of Convex Objects: The Method of Separating Axes. http://www.geometrictools.com/, 2008.

[4] Gottwald, D.; Kahl, G.; Likos, C. N. J. Chem. Phys. 2005, 122, 204503.

[5] Ni, R.; Gantapara, A. P.; de Graaf, J.; van Roij, R.; Dijkstra, M. Soft Matter 2012, 8, 8826.

[6] Frenkel, D.; Smit, B. Understanding Molecular Simulation, 2nd ed.; Academic Press, Inc.: Orlando, FL, USA, 2001.

[7] Widom, B. J. Chem. Phys. 1963, 39, 2808.

[8] Frenkel, D.; Ladd, A. J. C. J. Chem. Phys. 1984, 81, 3188.

[9] Noya, E. G.; Vega, C.; Doye, J. P. K.; Louis, A. A. The Journal of Chemical Physics 2007, 127, 054501.

[10] Fortini, A.; Dijkstra, M.; Schmidt, M.; Wessels, P. P. F. Phys. Rev. E 2005, 71, 051403.

[11] Donev, A. Jammed Packings of Hard Particles. Ph.D. thesis, Princeton University, 2006.
[12] Marechal, M.; Dijkstra, M. Phys. Rev. E 2010, 82, 031405 .

[13] Smallenburg, F.; Filion, L.; Marechal, M.; Dijkstra, M. Proc. Natl. Acad. Sci. USA 2012, 109, 17886.

[14] Minkowski, H. Nachr. K. Ges. Wiss. Göttingen 1904, $11,311$.

[15] Betke, U.; Henk, M. Comput. Geom. 2000, 16, 157.

[16] Filion, L.; Dijkstra, M. Phys. Rev. E 2009, 79, 046714.

[17] Damasceno, P. F.; Engel, M.; Glotzer, S. C. ACS Nano 2012, 6, 609 .

[18] Damasceno, P. F.; Engel, M.; Glotzer, S. C. Science 2012, 337, 453.

[19] Evers, W. H.; Friedrich, H.; Filion, L.; Dijkstra, M.; Vanmaekelbergh, D. Angew. Chem. Int. Ed. 2009, 48, 9655.

[20] Evers, W. H.; de Nijs, B.; Filion, L.; Castillo, S.; Dijkstra, M.; Vanmaekelbergh, D. Nano Lett. 2010, 10, 4235.

[21] Stokes, H. T.; Hatch, D. M.; Campbell, B. J. FindSym Software Package. http://stokes.byu.edu/isotropy. html, 2007.

[22] for Computational Materials Science, C. Database of molecular prototypes corresponding to the 230 existing space groups. http://cst-www.nrl.navy.mil/lattice/ index.html, 2008. 